\title{
,ance \\ A Low-Cost Smart Digital Mixer System Based on Speech Recognition
}

\author{
Shin-Chi Lai ${ }^{1}\left(\mathbb{D}\right.$, Ying-Hsiu Hung ${ }^{2}$, Yi-Chang Zhu ${ }^{3}$, Szu-Ting Wang ${ }^{3}$, Ming-Hwa Sheu ${ }^{2}$ and Wen-Ho Juang ${ }^{4, *}$ \\ 1 Department of Automation Engineering, National Formosa University, No. 64, Wunhua Rd., \\ Huwei Township, Yunlin 632, Taiwan; shivan0111@nfu.edu.tw \\ 2 Department of Electronic Engineering, National Yunlin University of Science and Technology, No. 123, Sec. 3, \\ Yunlin 632, Taiwan; wer850718906@gmail.com (Y.-H.H.); sheumh@yuntech.edu.tw (M.-H.S.) \\ 3 Doctor's Program of Smart Industry Technology Research and Design, National Formosa University, No. 64, \\ Wunhua Rd., Huwei Township, Yunlin 632, Taiwan; yichang.bs@gmail.com (Y.-C.Z.); \\ giniwang.nfu@gmail.com (S.-T.W.) \\ 4 Department of Computer Science and Information Engineering, National Formosa University, No. 64, \\ Wunhua Rd., Huwei Township, Yunlin 632, Taiwan \\ * Correspondence: riverjuang@nfu.edu.tw; Tel.: +886-5-2950955
}

check for updates

Citation: Lai, S.-C.; Hung, Y.-H.; Zhu, Y.-C.; Wang, S.-T.; Sheu, M.-H.; Juang, W.-H. A Low-Cost Smart Digital

Mixer System Based on Speech

Recognition. Electronics 2022, 11, 604. https://doi.org/10.3390/

electronics11040604

Academic Editor: Charles Tijus

Received: 31 December 2021

Accepted: 13 February 2022

Published: 16 February 2022

Publisher's Note: MDPI stays neutral with regard to jurisdictional claims in published maps and institutional affiliations.

Copyright: (C) 2022 by the authors. Licensee MDPI, Basel, Switzerland. This article is an open access article distributed under the terms and conditions of the Creative Commons Attribution (CC BY) license (https:// creativecommons.org/licenses/by/ $4.0 /)$.

\begin{abstract}
When a band is performing at a public occasion, certain sound effects are expected to be added to enliven the atmosphere. To achieve this effect, microphones and instruments are all connected to an audio mixer, then the expected audio output will be played through the speakers. However, sound engineers always spend plenty of time tuning the mixer until the satisfied results are obtained. This paper presents a smart digital mixer system that integrates touch control, speech control, and commonly used functions on an Android mobile platform to improve the mobility of audio mixer while tuning. The proposed system adopts a digital signal processor (DSP) as the core of the hardware architecture. The application provides a UI interface on an Android mobile phone in order to achieve the functions of speech recognition and touch control. The control commands will be transmitted to DSP via Bluetooth 5.0, self-defined Bluetooth packet format (SBPF), and data transfer controller (DTC). The main contribution of this work is to propose multiple functions of a mixer system with a convenient and interactive user interface. The experimental results show the average accuracy of all respondents reached $92.3 \%$. Moreover, the proposed system has the advantage of having a low-cost hardware circuit design, and provides high flexibility of setting for the audio mixer system according to the user's preference.
\end{abstract}

Keywords: mixer; digital signal processor; speech recognition; Bluetooth packet format; cyclic redundancy check; $\mathrm{I}^{2} \mathrm{C}$

\section{Introduction}

At large musical events, an audio mixer is used for tuning the results of the sounds by manually adjusting the knobs of the volume or equalizer. However, it takes lots of time to achieve the ideal situation precisely by operating manual knobs. A powerful audio mixer is an electronic device for combining and adjusting sounds of many different audio signals [1]. It cannot only modify the spectrum and amplitude of the input signal, but it also integrates various separate audio channels to output the audio signal. Compared with traditional analog mixers [2], digital mixers [3] can mix a variety of effects at the same time. It has advantages in terms of cleaner sound quality, higher signal-to-noise ratio noise suppression, and smaller device volume, but it has a more complicated operation.

In general, a sound engineer, who is responsible for the public address (PA) system, must satisfy and deal with all requests from performers, musicians, and listeners during a live band performance. While different instruments are played, performers will have different and individual adjustment needs. The traditional audio mixer has multiple input 
channels. Through the audio mixer, the audio input can be played via speakers [4]. It is often necessary for the sound engineer to listen to the audio effects on the spot and to then make adjustments immediately. It is not easy for an inexperienced person to control every detailed operation and the sound effects. To adjust the mixer system effectively, the integration of platform functions has gradually attracted attention. For example, Jaloudi's [5] intergraded wireless and wired microphone system transmits the audio input via a wired cable to the RCA connector on the mixing console. In addition, Yao [6] developed an interface that makes it easy to adjust the sound effect on the application. The audio output is transmitted to a Class-D amplifier via a wired cable. In addition, it is still a hard work for an experienced sound engineer to remotely control an audio mixer [7] and to make the auditory effects become more stunning. Therefore, some approaches have proposed a flexible way to use manual adjustment and control [1,8] on an audio mixer, or to reduce the difficulty of the mixer operation.

The proposed development platform provides remote audio control and speech recognition, which can connect the applications (APP) on mobile phones and the mixer via Bluetooth, so the sound engineer can walk around in front of the stage for testing. The sound engineer can adjust and change the parameters of setting in time to achieve the best output of audio. The proposed smart system platform used in this article is a prototype design to integrate various modules. The DSP kernel of the mixer is KT0707 [9], and the micro control unit (MCU) kernel of DTC is implemented by Atmeaga328p [10]. The selfdeveloped APP using the library of SpeechRecognizer [11] combines speech recognition and remote-control technologies, which allows us to upload the speech to the Google cloud server, and then this service returns the string data. It yields the maximized accuracy of speech control on the APP.

The problems encountered with the audio mixer and the proposed solutions are listed in Table 1. The proposed work can improve the drawbacks of the traditional audio mixer, i.e., labor-intensive, non-remote control [2-7], imprecise mixer knob tuning [2-5,7], and dense layout of the operation interface [4,5,8]. Further detail is introduced in Section 2. To compare with the products on the market, smart speakers with a similar price and functions [12-14] are employed in the experimental results. Although the smart speakers also provide application and functions of remote control and speech recognition, the equalizer and reverberation cannot be tuned appropriately. In this case, the user cannot achieve the best sound effect.

Table 1. Problems of audio mixers and the proposed solutions.

\begin{tabular}{cc}
\hline \multicolumn{1}{c}{ Problems in Use } & Proposed Solutions \\
\hline $\begin{array}{c}\text { The audio mixer is complicated to operate just } \\
\text { by one sound engineer. The manipulation is } \\
\text { labor intensive. }\end{array}$ & $\begin{array}{c}\text { The proposed smart audio mixer is based on } \\
\text { Bluetooth transmission to create a connection } \\
\text { between the audio mixer and application. }\end{array}$ \\
\hline $\begin{array}{c}\text { The traditional audio mixer does not possess a } \\
\text { wireless remote-control function. }\end{array}$ & $\begin{array}{c}\text { The remote-control function can be realized } \\
\text { based on Bluetooth technology, including } \\
\text { volume, reverberation, and equalizer tuning. }\end{array}$ \\
$\begin{array}{c}\text { The mixer knobs are hard to tune accurately, } \\
\text { and it is time-consuming to obtain a satisfying } \\
\text { sound effect. }\end{array}$ & $\begin{array}{c}\text { The digital signal processor is regarded as the } \\
\text { core in audio mixer, and tuning can be } \\
\text { operated with a precise digital scale. }\end{array}$ \\
$\begin{array}{c}\text { The layout of the operational interface on APP } \\
\text { is dense so as to develop full control functions. }\end{array}$ & $\begin{array}{c}\text { The function of speech recognition is included } \\
\text { in the application so that the user can control } \\
\text { the devices by speech in real time. }\end{array}$ \\
\hline
\end{tabular}

\section{Methods}

The structures of the proposed smart system platform design are divided into three parts: (1) digital mixer adjustment (DSP, KT0707), (2) DTC, and (3) Android APP design. Figure 1 shows the block diagram of the proposed system platform design. 


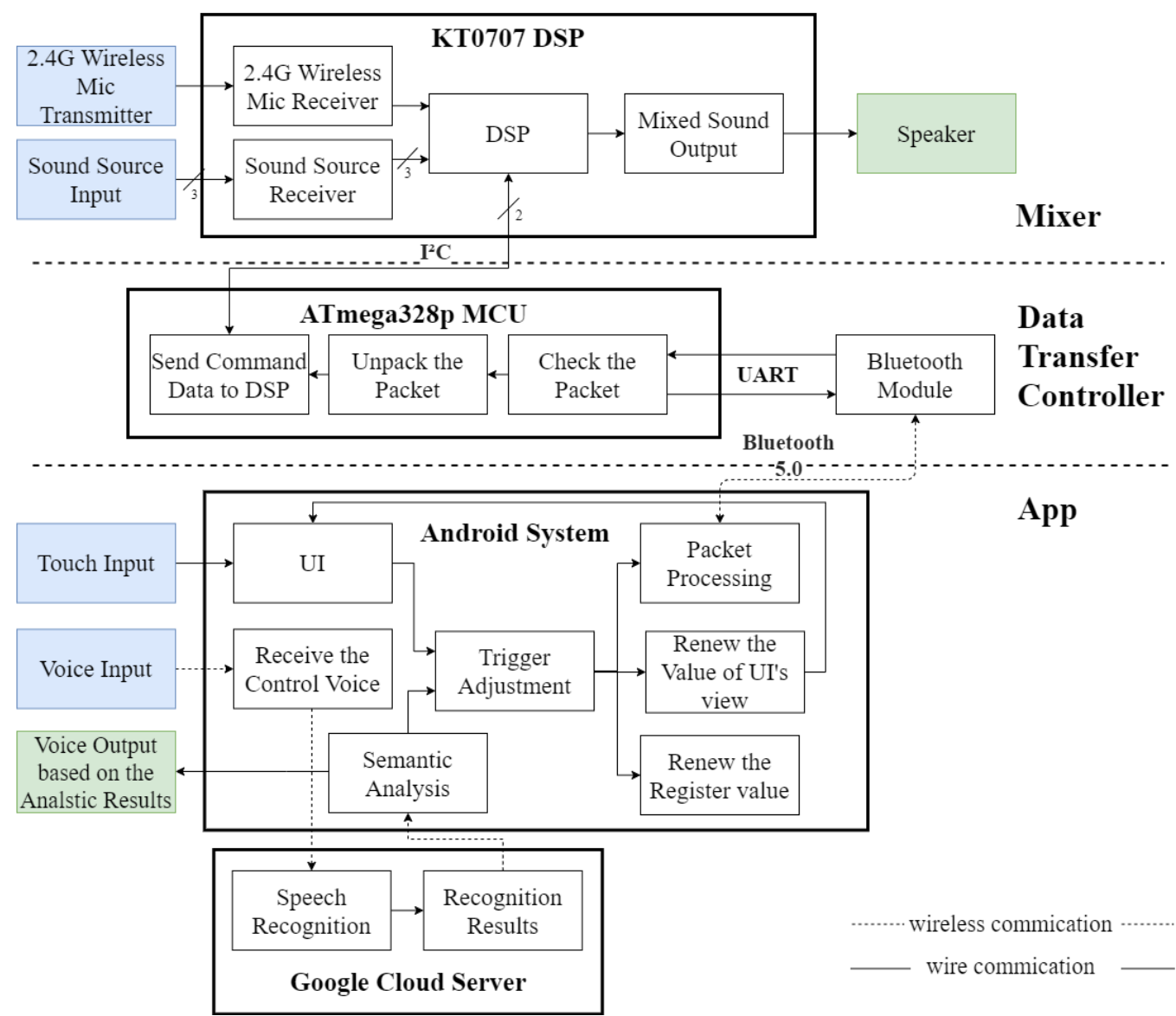

Figure 1. Proposed smart system platform designs for APP, DTC, and the mixer.

To satisfy the requests of the performers, KT0707 was deployed as the DSP of the proposed design, after several actual tests were implemented. KT0707 can simulate the performance of a small-scale band outdoors, i.e., three audio input channels are specified for keyboard, bass, and guitar individually, and a wireless microphone input channel is specified for the vocals. The audio signals in the wireless microphone and three-channel audio sources are transmitted to the mixer's analog digital converter (ADC) through radio frequency continuously. All the audio input processed by the audio mixer can be played via speakers. The DSP kernel of the audio mixer has a powerful digital processing ability that can deal with many control commands such as "volume tuning", "volume up by $10 \%$ ", and "adjustment of high/middle/low frequency" requested from the user on the APP. DTC receives the packets of control command via Bluetooth from the APP, and then it transmits the command into the DSP for further control by $\mathrm{I}^{2} \mathrm{C}$. In addition to the UI interface, the APP also provides a speech recognition function and a semantic recognition function to convert speech into text. The received semantic contents are mapped to the corresponding control codes, and all packets including the control codes are transmitted to DTC via Bluetooth.

\subsection{Proposed Data Transfer Controller Design}

2.1.1. Hardware Design

Atmeaga328p is utilized in MCU as a kernel of the DTC circuit. AMS1117-3.3v and AMS1117-5v [15] are both employed as the voltage suppliers for Bluetooth 5.0, KT0707 (DSP), and MCU. A 16-MHz quartz oscillator is used as the clock of MCU. The functions of the SPI library on the Arduino platform are deployed for the burning coded program on MCU [16]. The Bluetooth communication module [17] for the UART interface is used in the proposed design. In the design of the printed circuit board (PCB), copper is retained 
on the unused area and is connected to a ground for strengthening the stability of the Bluetooth transmission. The Bluetooth will be settled along the border of PCB to achieve better transmissions. Figure 2 demonstrates the proposed DTC circuit layout design with a size of $4.8 \times 4.2 \mathrm{~cm}^{2}$.

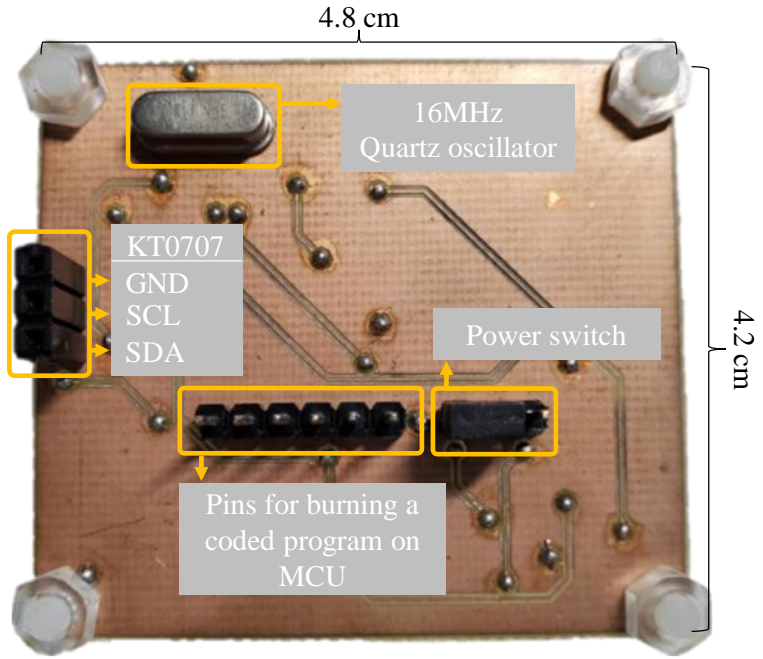

(a)

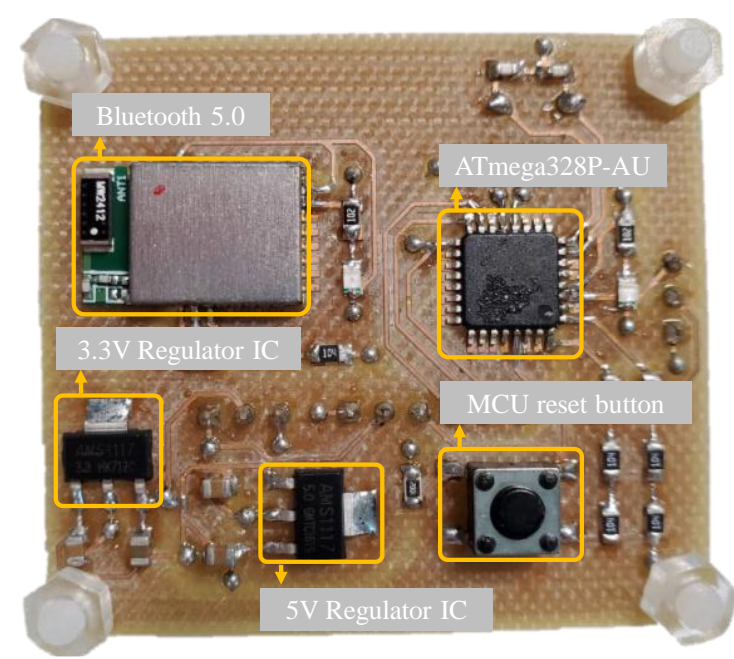

(b)

Figure 2. Proposed DTC circuit placement and layout design: (a) button view and (b) top view.

\subsubsection{Firmware Design}

The DTC is mainly used as a bridge between the DSP and APP on mobile phones for communication. The firmware design for transmissions and communications will be programmed according to different protocols. The $\mathrm{I}^{2} \mathrm{C}$ library [18] is programmed according to the provided timing diagram of KT0707, as shown in Figure 3. A self-defined Bluetooth packet format (SBPF), as shown in Figure 4, is designed based on the Bluetooth 5.0 transmission protocol [19].

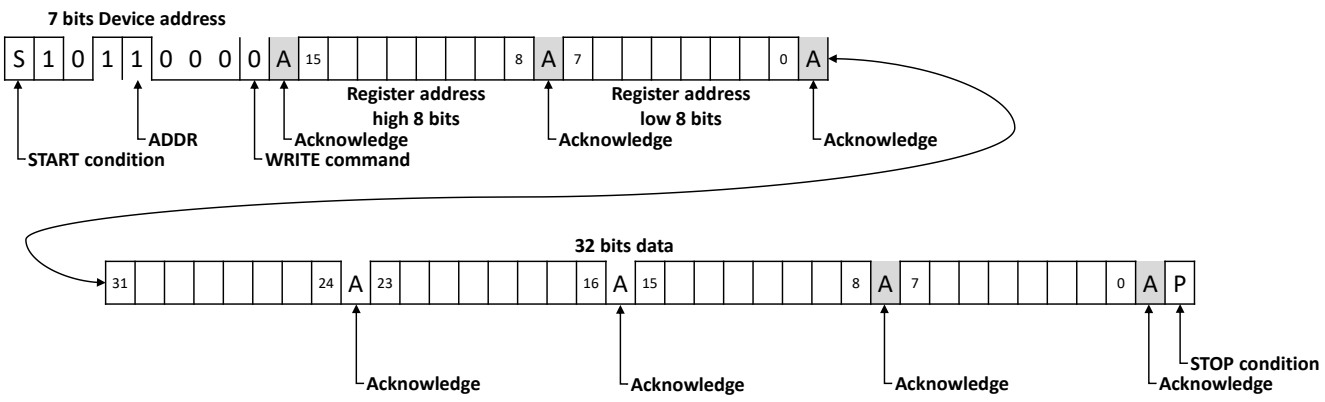

(a)
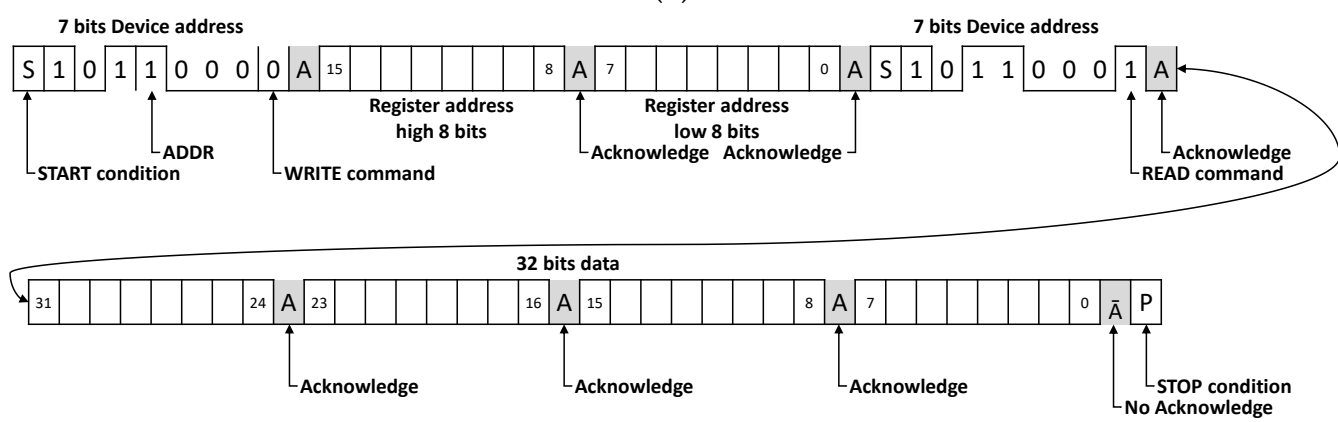

(b)

Figure 3. Timing diagram of $\mathrm{I}^{2} \mathrm{C}$ communication between MCU and DSP: (a) MCU and (b) MCU [9]. 


\begin{tabular}{|c|c|c|c|c|c|}
\hline 1 Byte & 1 Byte & 2 Bytes & 4 Bytes & 2 Bytes & 1 Byte \\
\hline $0 \times 10$ & $\begin{array}{l}\text { Packet } \\
\text { Length }\end{array}$ & $\begin{array}{l}\text { Register } \\
\text { Address }\end{array}$ & $\begin{array}{c}\text { Register } \\
\text { Value }\end{array}$ & CRC & $0 \times 0 F$ \\
\hline
\end{tabular}

(a)

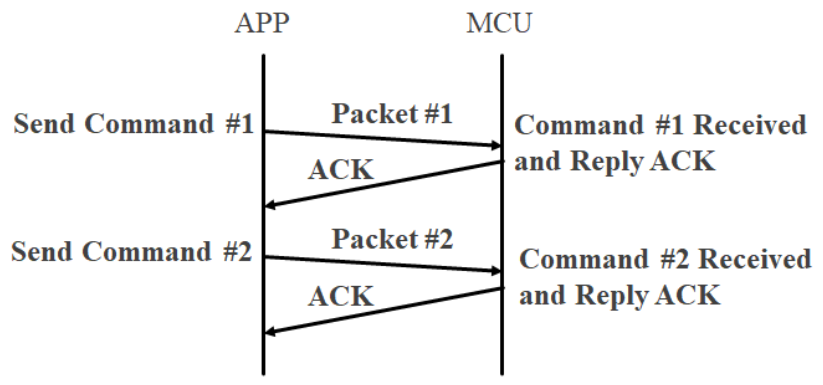

(b)

Figure 4. The proposed self-defined Bluetooth packet format and ACK mechanism: (a) SBPF and (b) schematic diagram of the handshake of the ACK mechanism.

Figure 4a represents the format of SBPF with a length of 11 bytes. One byte is used for the left edge of the block, another byte is used for the packet length. Two bytes are used for storing the register address, and four bytes are used for storing the register value. Another two bytes are used for CRC, and the last byte is used for the right edge of the block. In addition, the proposed SBPF adopts CRC (CRC-16-CCITT) [20] and ACK mechanisms to improve the accuracy and integrity of the transmitted data. Figure $4 \mathrm{~b}$ provides further descriptions of the communication protocol. An MCU is employed as a slave terminal, and the proposed APP is the master terminal. In practice, a specific library is created to control the proposed SBPF. There are three main functions in the library, i.e., "General_Tx", "General_Rx", and "Interrupt_Process", are all are used for processing with the following actions: (1) packaging and scheduling, (2) received data filtering and identifying, and (3) time scheduling and retransmission mechanism. To confirm the integrity of packet transmission, an ACK mechanism is deployed in the proposed design. If the ACK is successfully received by the MCU, it indicates that the current packet has been correctly transmitted; if it fails, the current packet will be retransmitted again. However, if the maximum amount of retransmission times is exceeded, the current packet is ignored in order to prevent delaying subsequent packets.

The control commands of DSP, including the register names, values, and features are partially listed in Table 2 . The proposed smart system platform mainly contains the following functions: (1) switches of volume control for four input sources, (2) switch of equalization (EQ; low, middle, and high frequency) and the gain adjustments for four input sources, and (3) reverberation processing of two input sources.

The reverberation adjustment is divided into the following two parts: (1) Reverberation of room size, which will simulate the room size of the space where the analog sound made is located. The smaller value represents the smaller room. (2) The reverberation level is the influence level of the reverberation, and a larger value causes a heavier space sense. On the other hand, EQ-frequency control adjusts the center frequency of the gain, and the value ranges between $15 \mathrm{~Hz}$ and 20,600 Hz. EQ-gain control adjusts the gain centered on the control frequency, and the value ranges between $-24 \mathrm{~dB}$ and $12 \mathrm{~dB}$. 
Table 2. Partial function of the control registers in DSP (KT0707).

\begin{tabular}{|c|c|c|c|}
\hline Name & Address & Default Value & Feature Description \\
\hline AudioPath & $0 \times 0000$ & 0x00000701 & ADC0 3's volume switch \\
\hline ADC_PreVol & 0x0002 & $0 \times 67676767$ & ADC0 3's Volume control \\
\hline ReverbCtrl0 & 0x000D & 0x0B6CFEFC & ADC0, 1's reverb switch and reverb-room size control \\
\hline ReverbCtrl1 & 0x000E & 0x80485067 & $\mathrm{ADC} 0,1^{\prime}$ s reverb-level control \\
\hline EQ4Mic01_LowBand_Ctr10 & 0x004E & 0x003F02C3 & ADC $0,1^{\prime}$ s low frequency EQ-frequency control \\
\hline EQ4Mic01_LowBand_Ctrl1 & $0 \times 004 \mathrm{~F}$ & 0x00000000 & ADC $0,1^{\prime}$ s low frequency EQ-gain control, EQ switch \\
\hline EQ4Mic01_MidBand_Ctrl0 & 0x0050 & 0x006402C3 & ADC $0,1^{\prime}$ s middle frequency EQ-frequency control \\
\hline EQ4Mic01_MidBand_Ctrl1 & 0x0051 & 0x00000000 & ADC $0,1^{\prime}$ s middle frequency EQ-gain control, EQ switch \\
\hline EQ4Mic01_HighBand_Ctrl0 & 0x0052 & 0x00A002C3 & ADC $0,1^{\prime}$ s high frequency EQ-frequency control \\
\hline EQ4Mic01_HighBand_Ctrl1 & $0 \times 0053$ & 0x00000000 & ADC 0, 1's high frequency EQ-gain control, EQ switch \\
\hline EQ4Ext01_LowBand_Ctr10 & 0x008A & 0x003F02C3 & ADC 2, 3's low frequency EQ-frequency control \\
\hline EQ4Ext01_LowBand_Ctrl1 & 0x008B & 0x00000000 & ADC 2, 3's low frequency EQ-gain control, EQ switch \\
\hline EQ4Ext01_MidBand_Ctr10 & 0x009A & 0x09C402C3 & ADC 2, 3's middle frequency EQ-frequency control \\
\hline EQ4Ext01_MidBand_Ctrl1 & 0x009B & 0x00000000 & ADC 2, 3's middle frequency EQ-gain control, EQ switch \\
\hline EQ4Ext01_HighBand_Ctrl0 & 0x009E & $0 \times 189 \mathrm{C} 02 \mathrm{C} 3$ & ADC $2,3^{\prime}$ s high frequency EQ-frequency control \\
\hline EQ4Ext01_HighBand_Ctrl1 & 0x009F & 0x00000000 & ADC 2, 3's high frequency EQ-gain control, EQ switch \\
\hline
\end{tabular}

\subsection{Proposed APP Design}

APP design is the core of the proposed system. The complete APP functional block diagram is shown in Figure 5. As multi-thread technology is employed in APP, the UI interaction and speech recognition can be executed simultaneously. Different from the MCU, single thread technology only can execute single events to prevent memory conflicts. Except for the above-mentioned functions, the Bluetooth transmission protocol adopts Bluetooth low energy (BLE) technology in the APP to program the connection and transmission functions. It can reduce the communication delay of $\mathrm{MCU}$ and the consumption of the smart system platform. In addition, the proposed APP includes functions of speech recognition, semantic analysis, and the storage of DSP instruction sets.

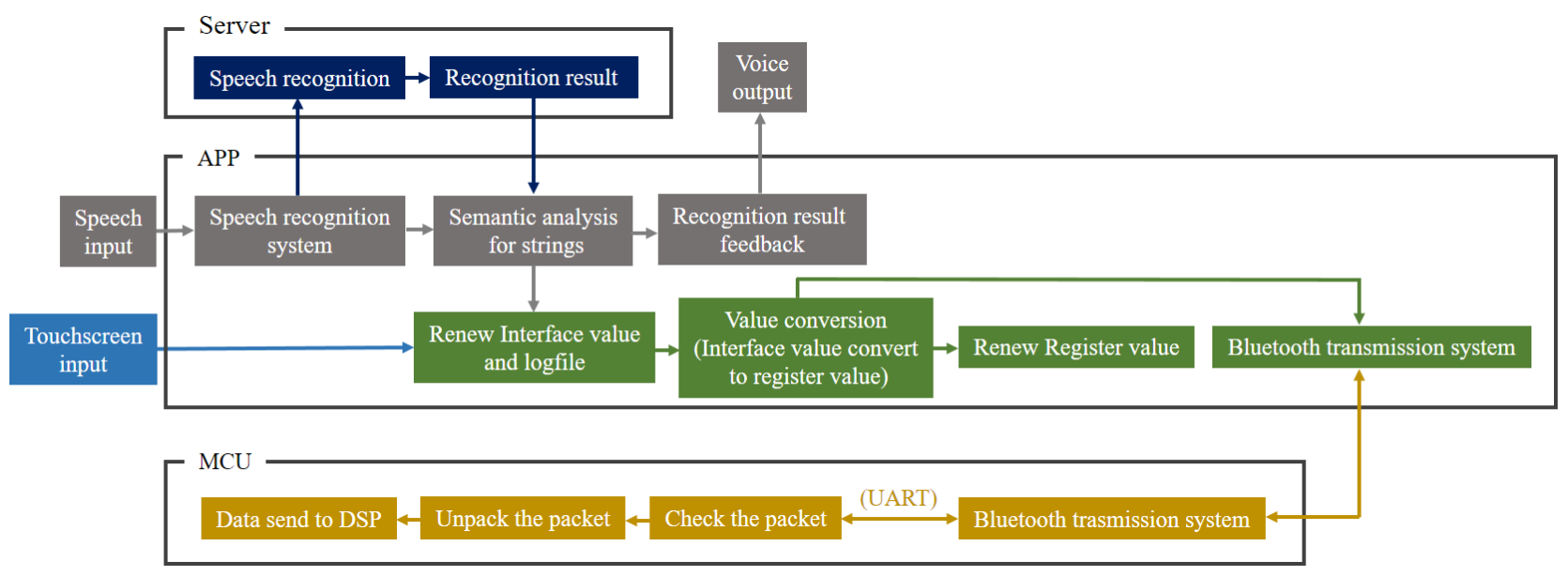

Figure 5. APP function block for the proposed smart digital mixer system.

In this work, speech recognition technology provided on the Android platform by Google SpeechRecognizer [21], which is employed to realize the function of speech control. This provides the functions to convert speech into text with a high accuracy. Additionally, 
it has the advantage of being able to be executed in various noisy environments. The recognized control command will be converted into text. The function and the scale in the text will be analyzed by semantic analysis, then the packets will be transmitted by DSP to tune the mixer. For example, if the recognized control command is "turn on the volume", it will be recognized that the volume needs to be tuned and the switch will be turned on. The pseudo code of speech recognition is shown in Figure 6.

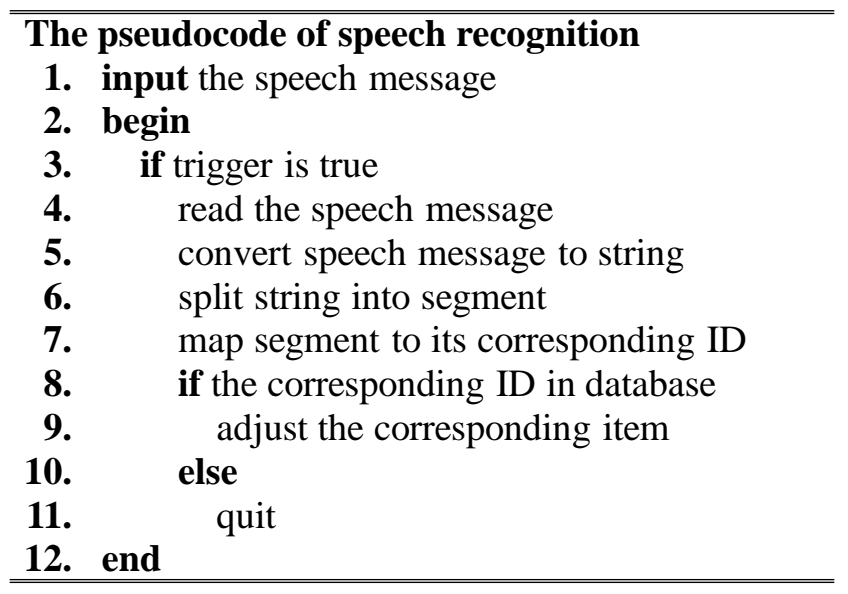

Figure 6. The pseudocode of speech recognition.

The main control program of SBPF on the APP, which is named BEPAPP, is a selfdevelopment programmed in the proposed system. The program on the MCU will receive, analyze, and respond to the packets. Here, we use $\mathrm{C}++$ on MCU, and JAVA is used for APP. Furthermore, the interrupt mechanism will be triggered on MCU as it applies a single thread model. In contrast, multiple threads can be applied on an Android system, which can improve overall performance.

The flowcharts of the proposed smart digital mixer system, including speech recognition, UI interface, and Bluetooth, are shown in Figure 7. The right flowchart in Figure 7 describes the processing of the Bluetooth transmission. On the APP, "scan to peripheral devices" can be triggered, and the connection can be created between Bluetooth and the audio mixer. If the connection has been created successfully, the user can enter the main operation interface. The middle flowchart in Figure 7 describes the processing of the user interface, and the control command can be obtained according to the speech recognition. The component ID is detected and triggered according to the control command. The left flowchart in Figure 7 describes the processing of speech recognition. Speech recognition is triggered once the voice is received on the APP, and then the content of the speech is converted to text. Next, semantic analysis is employed to obtain the corresponding control command. Finally, the control command is transmitted to MCU via SBPF and Bluetooth. 


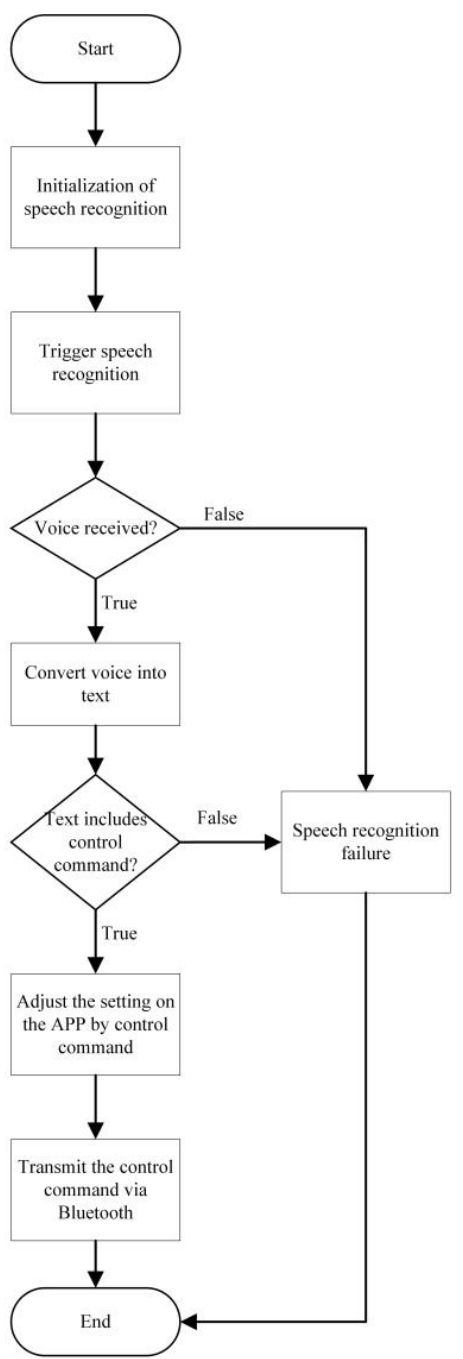

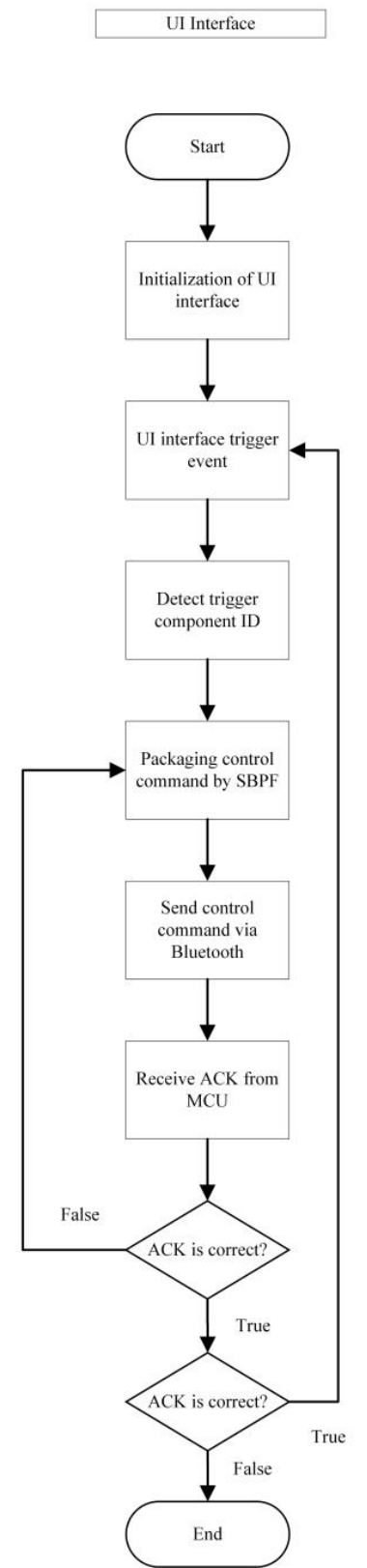

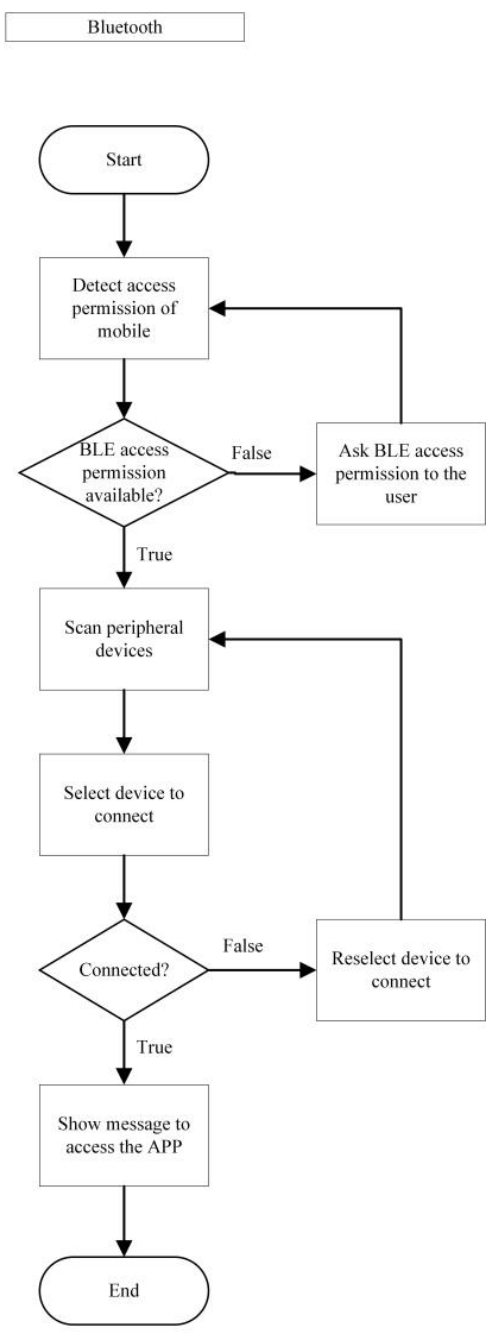

Figure 7. Flowcharts of the proposed smart digital mixer system including speech recognition, UI interface, and Bluetooth.

\section{Realization Results, Comparison, and Discussion}

For the interface of the APP, ConstraintLayout [22] provided by the Android platform is used as the base layout method. The operation process of the APP is introduced in Figure 8. The initial screen of the interface is shown in Figure 8a. Bluetooth scanning will be activated when the button "start scanning" is pressed. All the devices that can be connected will be shown in the list. Once the Bluetooth device is connected, then a screen will be shown, as in Figure 8b. Press the "setting" button to enter the main screen of the control functions. The main control screen is shown in Figure 8d. The tuning button of the main volume is located in the middle of the screen. The volume control screen is shown in Figure $8 \mathrm{c}$. The slider bar is used to control the volume adjustment of the wired/wireless microphone, external sound source volume, reverberation level, and the room size. The equalizer control screen is shown in Figure 8e. The slider bar is used to control the gain of the high, medium, and low frequency band for the frequency response of the input audio. 
The hardware framework of the proposed system is demonstrated in Figure 9. The user can manipulate the mixer system on the APP. Once the devices are connected via Bluetooth, audio tuning can be executed sing voice control or touch control. The proposed design improves the drawbacks of the traditional audio mixer system to a smart system with intuitive operation and rapid response.

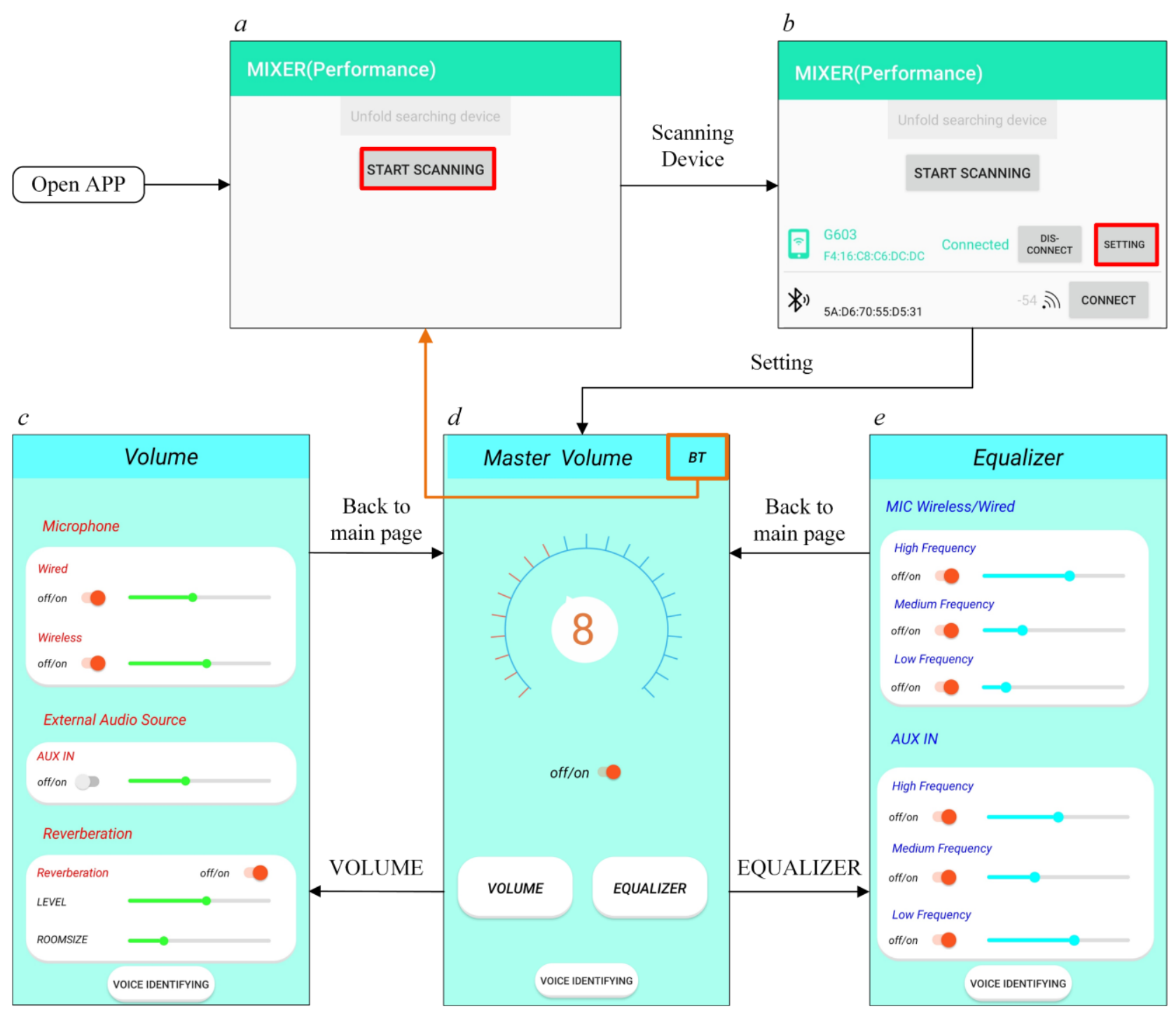

Figure 8. Flowchart of the APP operation.

Table 3 summarizes the comparison results of seven items for common commercial products of smart speakers $[7,8,12-14]$. As the price is one of the consider factors, the total cost of the proposed design was USD 398.95 dollars, which included a JBL PartyBox 300 speaker (USD 379.95 dollars) and smart digital mixer system (USD 19 dollars). 


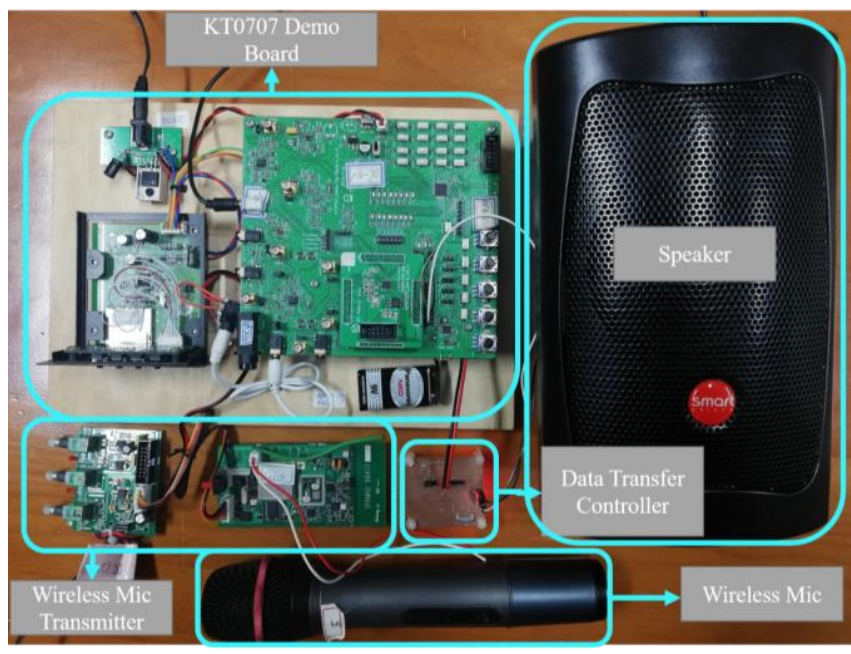

(a)

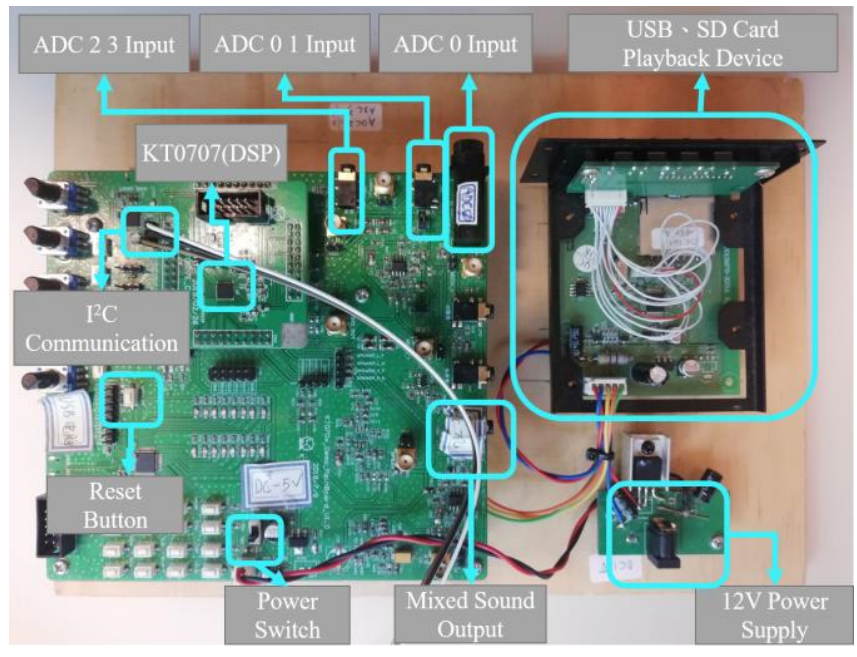

(b)

Figure 9. The actual prototype design of the proposed system: (a) complete hardware and (b) KT0707 demo board.

Table 3. A comparison between commercial smart speakers, mixers, and the proposed design.

\begin{tabular}{ccccccc}
\hline Item & [7] & [8] & [12] & [13] & [14] & Proposed \\
\hline Voice control & N/A & N/A & $\checkmark$ & $\checkmark$ & $\checkmark$ & $\checkmark$ \\
\hline Touch control & $\checkmark$ & $\checkmark$ & $\checkmark$ & $\checkmark$ & $\checkmark$ & $\checkmark$ \\
\hline Wireless control (APP) & N/A & N/A & $\checkmark$ & $\checkmark$ & $\checkmark$ & $\checkmark$ \\
\hline Mixer type & Digital & Analog & N/A & N/A & N/A & Digital \\
\hline Equalizer control & $\checkmark$ & $\checkmark$ & N/A & N/A & N/A & $\checkmark$ \\
\hline Reverb control & $\checkmark$ & $\checkmark$ & N/A & N/A & N/A & $\checkmark$ \\
\hline Bluetooth connection & N/A & $\checkmark$ & $\checkmark$ & $\checkmark$ & $\checkmark$ & $\checkmark$ \\
\hline Amplifier and speaker & N/A & N/A & $\checkmark$ & $\checkmark$ & $\checkmark$ & $\checkmark$ \\
\hline Price (USD) & 253 & - & 50 & 50 & 299 & $19^{1}$ \\
\hline 1
\end{tabular}

${ }^{1}$ Smart digital mixer system. ${ }^{2}$ JBL PartyBox 300 speaker.

The proposed low-cost smart digital mixer system includes an application (APP), data transfer controller (DTC), and digital signal processor (DSP). DSP can be connected to diverse speaker output devices with a $3.5 \mathrm{~mm}$ AUX cable, and it is not limited to the speaker used, which is a JBL PartyBox 300 speaker in the article. The functions of most of the products on the market are bundled on the speakers. The different levels of speaker devices have different sound qualities. Practical testing reveals that the sound quality is premium and does not have noticeable distortion by playing the audio via wired headphones. In addition, the output sound quality depends on digital signal processing, speakers, and the employed Class-D audio amplifier. In general, a Class-D audio amplifier costs about 1 to 50 US dollars depending on the different power output and output channels. The main contribution of the proposed work is to provide a DSP platform design that can achieve adjustment of the audio mixer, and the sound effect can be transmitted to alternative output devices by APP control with speech recognition. The JBL PartyBox 300 speaker is adopted in the experiments, and its cost also is included in Table 3. Although some companies develop APPs for their own products to achieve remote-control [12-14], the proposed system still contains all the functions of a smart speaker. The users can adjust the effects of the equalizer and reverberation according to their preference. Compared to the analog 
mixer, which only can achieve one adjustment at a time, the digital mixer can achieve multiple adjustments at one time. Therefore, the proposed system of the digital mixer has more mobility than the analog mixer. Overall, the proposed system has the advantage of a low-cost price and having full functions. It also provides high flexibility to connect with multiple types of Bluetooth speakers.

To verify the accuracy of speech recognition on the APP, 14 control commands of speech were created in the proposed system. The 13 eligible respondents (R1-R13) whose ages were between 20 and 30 were invited to test the speech recognition function. Every control command was tested at least three to five times. The total tests reached 770 times. The testing environments were set up in office and band rehearsal studios. The test result is shown in Figure 10. The accuracy reached $90 \%$ of nine respondents. The average accuracy of all respondents reached $92.3 \%$. The result revealed that the accuracy of speech recognition achieved excellent results, even though the testing environment had ambient noise.

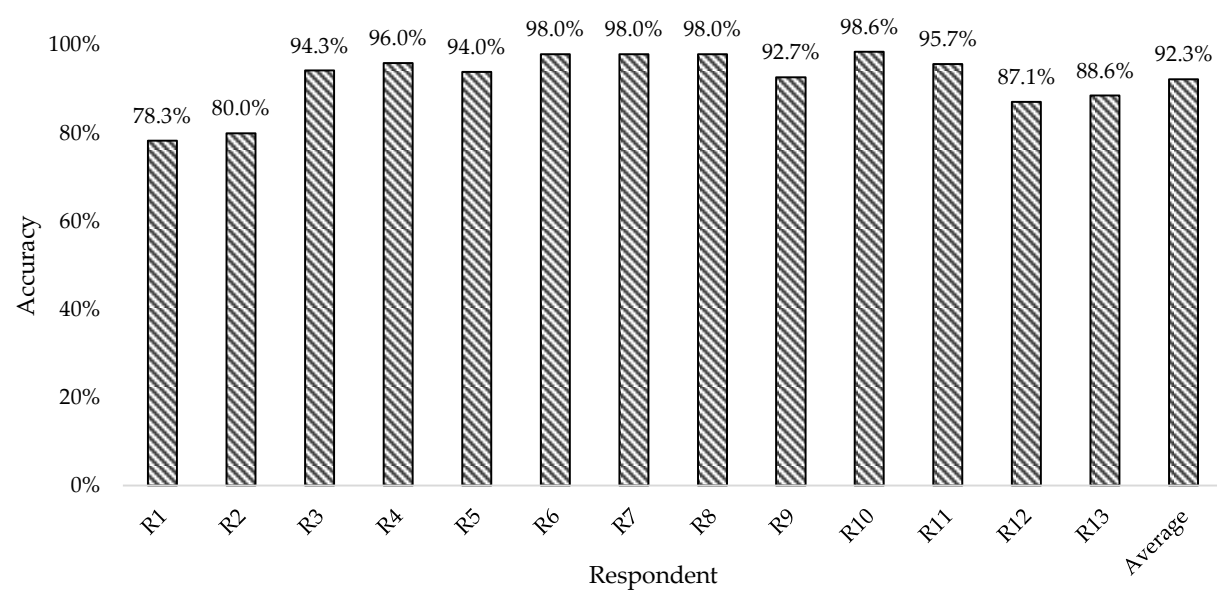

Figure 10. Accuracy of speech recognition of different respondents.

\section{Conclusions}

Overall, the proposed smart digital mixer system can control the devices remotely via speech on the APP. The convenient design can reduce the manpower of sound engineers and the rehearsal time, especially at large-scale musical events. Thirteen respondents were invited to test the speech recognition on the APP, and the total test times were over 770 times. The results show the average accuracy of all respondents reached $92.3 \%$. Speech recognition can resist little noise, but there will be recognition errors when the environment has ambient noise. Based on previous experience, headphones with active noise cancellation can be applied to improve the problem. Moreover, this low-cost digital system design could potentially become the solution to replace commercial products in the future.

Author Contributions: Conceptualization, S.-C.L. and W.-H.J.; methodology, S.-C.L. and W.-H.J.; software, Y.-H.H. and Y.-C.Z.; validation, Y.-H.H. and Y.-C.Z.; formal analysis, S.-C.L. and M.-H.S.; investigation, S.-C.L., Y.-H.H. and Y.-C.Z.; resources, S.-C.L. and W.-H.J.; data curation, Y.-H.H. and Y.-C.Z.; writing-original draft preparation, Y.-H.H., Y.-C.Z. and S.-T.W.; writing-review and editing, S.-C.L., Y.-H.H. and S.-T.W.; visualization: Y.-H.H. and Y.-C.Z.; supervision: S.-C.L. and M.-H.S.; project administration, S.-C.L.; funding acquisition, S.-C.L. and W.-H.J. All authors have read and agreed to the published version of the manuscript.

Funding: This work was supported by the Ministry of Science and Technology, Taiwan, under grants MOST 110-2221-E-150-045 and MOST 109-2221-E-150-043, and was also supported by the Smart Machinery and Intelligent Manufacturing Research Center, National Formosa University, Yunlin, Taiwan, under grants 111IT100-D.

Acknowledgments: The authors would like to thank Yu-Hsiu Chang, Yi-Zhen Chen, Yong-Jyun Wang, and Pei-Wei Yu for their kind help on this research topic. The authors also would like to thank CHIAYO Electronics for their valuable suggestions and support for the system integration. 
Conflicts of Interest: The authors declare no conflict of interest.

\section{References}

1. Marcu, A.E.; Vlădescu, M.; Dobre, R.A. Cost Effective Remote Control System for Analog Audio Mixers. In Proceedings of the 2018 IEEE 24th International Symposium for Design and Technology in Electronic Packaging (SIITME), Iasi, Romania, 25-28 October 2018; pp. 280-283.

2. Dugan, D. Tutorial: Application of Automatic Mixing Techniques to Audio Consoles. SMPTE J. 1992, 101, 19-27. [CrossRef]

3. Uskokovic, M.; Ivancevic, B. The implementation of digital audio processors in analog multimedia audio systems. In Proceedings of the 17th International Conference on Applied Electromagnetics and Communications, Dubrovnik, Croatia, 1-3 October 2003; pp. 131-134.

4. Branco, D.P.; Skliarova, I.; Vieira, J. Reconfigurable digital audio mixer for electroacoustic music. In Proceedings of the 2010 International Conference on Reconfigurable Computing and FPGAs, Cancun, Mexico, 13-15 December 2010; pp. $132-137$.

5. Jaloudi, S. Software-defined radio for modular audio mixers: Making use of market-available audio consoles and software-defined radio to build multiparty audio-mixing systems. IEEE Consum. Electron. Mag. 2017, 6, 97-104. [CrossRef]

6. Yao, S.N. Audio Effect Units in Mobile Devices for Electric Musical Instruments. IEEE Access 2019, 7, 138-145. [CrossRef]

7. CHIAYO_SMART 300 Personal Wireless PA System. Available online: http:/ /www.chiayo.com.tw/english/smart.html (accessed on 23 August 2021).

8. Anghel, F.V.; Marcu, A.E.; Dobre, R.A.; Drăgulinescu, A.M.C. Improved Remote Control System for Analog Audio Mixers Featuring Internet of Things Elements. In Future Access Enablers for Ubiquitous and Intelligent Infrastructures; Springer: Cham, Germany, 2019; pp. 138-145.

9. KG Technologies. 32-Bits Audio Processor with Four ADCs and Three DACs, KT0707 Datasheet; Revised April 2018; KG Technologies: Rohnert Park, CA, USA, 2017.

10. Atmel. 8-Bit Microcontroller with 4/8/16/32 Kbytes In-System Programmable Flash. Atmel-8271 JS-AVR-ATmega-Datasheet_11; Atmel: San Jose, CA, USA, 2015.

11. Speechmatics API Documentation. Available online: https:/ / APP.speechmatics.com/api-details (accessed on 10 May 2020).

12. Echo Dot (3rd Gen)—Smart speaker with Alexa-Charcoal. Available online: https://www.amazon.com/Echo-Dot-3rd-GenBluetooth/dp/B08F8RQ469 (accessed on 23 August 2021).

13. Nest Mini_Google Store. Available online: https://store.google.com/tw/product/google_nest_mini_specs (accessed on 23 August 2021).

14. BEST BUY-Apple-HomePod-Space Gray. Available online: https://www.bestbuy.com/site/apple-homepod-space-gray/5902 410.p?skuId=5902410\&intl=nosplash (accessed on 23 August 2021).

15. ASM1117 1A Low Dropout Voltage Regulator. Available online: http://www.advanced-monolithic.com/pdf/ds1117.pdf (accessed on 5 August 2021).

16. Arduino-SPI. Available online: https://www.arduino.cc/en/reference/SPI (accessed on 26 August 2021).

17. Raytac Corporation. Bluetooth Low Energy Module, MDBT42-P512KV2 Approval Sheet; Raytac Corporation: New Taipei, Taiwan, 2021.

18. Wiki-I2C: Inter-Integrated Circuit. Available online: http://wiki.csie.ncku.edu.tw/embedded/I2C (accessed on 20 August 2021).

19. Bluetooth 5 Speed: How to Achieve Maximum Throughput for Your BLE Application. Available online: https://www.novelbits. io/bluetooth-5-speed-maximum-throughput/ (accessed on 18 June 2020).

20. Cyclic Redundancy Check-Wikipedia. Available online: https://en.wikipedia.org/wiki/Cyclic_redundancy_check (accessed on 26 August 2021).

21. SpeechRecognizer / Android Developers. Available online: https://developer.android.com/reference/android/speech/ SpeechRecognizer (accessed on 26 August 2021).

22. ConstraintLayout I Android Developers. Available online: https://developer.android.com/reference/androidx/constraintlayout/ widget/ConstraintLayout (accessed on 26 August 2021). 